\title{
A decrease in hepatic microRNA-9 expression impairs gluconeogenesis by targeting FOXO1 in obese mice
}

\author{
Caifeng Yan ${ }^{1,2} \cdot$ Jinfeng Chen ${ }^{3} \cdot{\mathrm{Min} \mathrm{Li}^{2} \cdot \text { Wenying Xuan }}^{4} \cdot \mathrm{Dongming} \mathrm{Su}^{4} \cdot \mathrm{Hui} \mathrm{You}^{2}$ • \\ Yujie Huang ${ }^{2} \cdot$ Nuoqi Chen ${ }^{3} \cdot$ Xiubin Liang ${ }^{2}$
}

Received: 2 December 2015 / Accepted: 29 February 2016/Published online: 22 March 2016

(C) Springer-Verlag Berlin Heidelberg 2016

\begin{abstract}
Aim/hypothesis MicroRNA-9 (miR-9) is involved in the regulation of pancreatic beta cell function. However, its role in gluconeogenesis is still unclear. Our objective was to investigate the role of miR-9 in hepatic glucose production (HGP). Methods MiR-9 expression was measured in livers of high-fat diet (HFD) mice and ob/ob mice. The methylation status of the miR-9-3 promoter regions in hepatocytes was determined by the methylation-specific PCR procedure. The binding activity of DNA methyltransferase (DNMT)1, DNMT3a and DNMT3b on the miR-9-3 promoter was detected by chromatin immunoprecipitation (ChIP) and quantitative real-time PCR assays. HGP was evaluated in vitro and in vivo. Glucose tolerance, insulin tolerance and pyruvate tolerance tests were also performed.

Results Reduced miR-9 expression and hypermethylation of the miR-9-3 promoter were observed in the livers of obese
\end{abstract}

Caifeng Yan, Jinfeng Chen and Min Li contributed equally to this study.

Electronic supplementary material The online version of this article (doi:10.1007/s00125-016-3932-5) contains peer-reviewed but unedited supplementary material, which is available to authorised users.

Xiubin Liang

liangxiubin@njmu.edu.cn

1 Department of Endocrinology, Clinical Medical College of Yangzhou University, Yangzhou, China

2 Department of Pathophysiology, Jiangsu Key Laboratory of Xenotransplantation, Nanjing Medical University,

Nanjing 210029, Jiangsu, China

3 Department of Endocrinology, Zhangzhou Affiliated Hospital of Fujian Medical University, Zhangzhou, China

4 Center of Pathology and Clinical Laboratory, Mingde Hospital of Nanjing Medical University, Nanjing, China mice. Further study showed that the binding of DNMT1, but not of DNMT3a and DNMT3b, to the miR-9-3 promoter was increased in hepatocytes from $o b / o b$ mice. Knockdown of DNMT1 alleviated the decrease in hepatic miR-9 expression in vivo and in vitro. Overexpression of hepatic miR-9 improved insulin sensitivity in obese mice and inhibited HGP. In addition, deletion of hepatic miR-9 led to an increase in random and fasting blood glucose levels in lean mice. Importantly, silenced forkhead box O1 (FOXO1) expression reversed the gluconeogenesis and glucose production in hepatocytes induced by miR-9 deletion.

Conclusions/interpretation Our observations suggest that the decrease in miR-9 expression contributes to an inappropriately activated gluconeogenesis in obese mice.

Keywords DNA methylation · FOXO1 · Insulin resistance · MicroRNA-9

$\begin{array}{ll}\text { Abbreviations } \\ \text { ChIP } & \text { Chromatin immunoprecipitation } \\ \text { ChIP-qPCR } & \text { Chromatin immunoprecipitation } \\ & \text { and quantitative real-time PCR } \\ \text { DMSO } & \text { Dimethylsulphoxide } \\ \text { DNMT } & \text { DNA methyltransferase } \\ \text { FOXO1 } & \text { Forkhead box O1 } \\ \text { G6Pase } & \text { Glucose-6-phosphatase, catalytic } \\ \text { GTT } & \text { Glucose tolerance test } \\ \text { HFD } & \text { High-fat diet } \\ \text { HGP } & \text { Hepatic glucose production } \\ \text { IR } & \text { Insulin resistance } \\ \text { ITT } & \text { Insulin tolerance test } \\ \text { miRs } & \text { MicroRNAs } \\ \text { MSP } & \text { Methylation-specific PCR } \\ \text { NC } & \text { Normal control }\end{array}$




$\begin{array}{ll}\text { NCD } & \text { Normal chow diet } \\ \text { PTT } & \text { Pyruvate tolerance test } \\ \text { qMSP } & \text { Quantitative methylation-specific PCR } \\ \text { si-NC } & \text { Normal control small interfering RNA } \\ \text { siRNA } & \text { Small interfering RNA } \\ \text { TSA } & \text { Trichostatin A }\end{array}$

\section{Introduction}

The liver is a key organ for maintaining glucose homeostasis in the body through insulin- and other metabolic hormonemediated events $[1,2]$. However, in diabetic patients with insulin resistance (IR), insulin fails to effectively inhibit gluconeogenic pathways, which results in an increase in hepatic glucose production (HGP) [3]. In addition, inappropriately elevated glucagon also increases HGP by promoting the expression and activity of gluconeogenic enzymes [4]. Therefore, elucidation of the underlying molecular mechanism of gluconeogenesis as well as HGP is of potential benefit for the development of effective therapies for the treatment of IR and type 2 diabetes.

The transcription factor forkhead box O1 (FOXO1) is implicated in multiple metabolic pathways [5]. In the liver, FOXO1 promotes HGP and activates the transcription of gluconeogenic enzymes, such as glucose-6-phosphatase (G6Pase) and PEPCK [6, 7]. Increased FOXO1 expression was observed in patients with hepatic IR and nuclear FOXO1 was significantly accumulated in the livers of highfat diet (HFD) mice and $d b / d b$ mice $[8,9]$. Further study demonstrated that overexpression of FOXO1 led to an increase in gluconeogenesis and HGP [10]. In contrast, deletion of hepatic FOXO1 in diabetic mice improved insulin sensitivity and glucose tolerance $[11,12]$. These studies indicated that FOXO1 acts as a regulator and mediator in the development of IR and type 2 diabetes [13], but the mechanism by which molecular factors regulate hepatic FOXO1 expression and activity during IR remains elusive.

MicroRNAs (miRs), a class of small non-coding RNAs, regulate target gene expression at the post-transcriptional level [14]. Growing evidence demonstrates that aberrant expression of miRs contributes to a variety of human diseases, including diabetes [15]. Recently, some studies have revealed a few miRs, notably miR-29a-c and miR-23a, are critical for gluconeogenesis $[16,17]$. Li demonstrated that miR-214 could suppress gluconeogenesis by directly targeting activating transcription factor 4 (ATF4), which affected FOXO1 transcriptional activity [18]. One particular miR, miR-9, has been discovered to be involved in the development of type 2 diabetes, based on evidence that miR-9 plays an important role in the regulation of in vitro and in vivo insulin secretion $[19,20]$. However, the effect of miR-9 on IR has not yet been investigated. Interestingly, FOXO1 has been confirmed as the direct target of miR-9 in haematopoietic cells [21]. Consequently, miR-9 might be involved in the regulation of insulin sensitivity in diabetes mellitus.

Recent research has shown that epigenetic DNA modifications contribute to the development of type 2 diabetes [22, 23]. Environmental factors, such as excess nutrition, can affect epigenetic regulation [24]. One of the major epigenetic mechanisms is DNA methylation, which has also been shown to be important in insulin sensitivity $[25,26]$. DNA methylation leads to a decrease in gene transcription by inhibiting the binding of transcriptional factors to gene promoters [27]. We have found that miR-9 is significantly downregulated in the liver of HFD mice and $o b / o b$ mice. We also found specific inhibition of DNA methylation could upregulate miR-9 expression in hepatocytes. We therefore hypothesised that epigenetic regulation might be involved in the regulation of miR9 expression in the livers of obese mice. We tested this hypothesis using HepG2 cells, primary hepatocytes, HFD-fed mice and $o b / o b$ mice.

\section{Methods}

Reagents See electronic supplementary material (ESM) Methods.

Cell culture The human hepatoblastoma cell line HepG2 was purchased from ATCC (Rockville, MD, USA) and cultured in DMEM supplemented with $10 \%$ (vol./vol.) FBS. HepG2 cells were cultured at $37^{\circ} \mathrm{C}$ in a humidified atmosphere containing $95 \%$ air and $5 \% \mathrm{CO}_{2}$. All cells were verified in our laboratory and there was no mycoplasma contamination.

Primary isolation and culture of hepatocytes Hepatocytes were isolated and cultured from the livers of male C57BL/6 J mice as described previously [28]. For further details, please refer to the ESM Methods.

Animal studies All animal studies were performed following the guideline established by the Research Animal Care Committee of Nanjing Medical University (Nanjing, China). Male C57BL/6J mice and 8- to 10-week-old male $o b /+$ or $o b /$ $o b$ mice were purchased from the Model Animal Research Center of Nanjing University (Nanjing, China). Diet-induced obese mice were fed ad libitum an HFD (D12492; Research Diets, New Brunswick, NJ, USA) for 15 weeks. Mice were housed under 12/12 h light/dark cycles with free access to food and water. $\mathrm{C} 57 \mathrm{BL} / 6 \mathrm{~J}$ mice were injected with normal control (NC) or miR-9 inhibitor, and $o b / o b$ mice were injected with $\mathrm{NC}$ or miR-9 mimic when they were 8 weeks old. HFD-fed mice were injected with $\mathrm{NC}$ or miR-9 mimic when they were fed an HFD for 11 weeks. Mice were injected i.v. through the tail vein with a lipid nanoparticle-formulated miR-9 inhibitor or miR-9 
mimic, at a dose of $100 \mathrm{nmol}$ in $0.2 \mathrm{ml}$ PBS, as described previously [29]. The DNA methyltransferase (DNMT) inhibitor was injected into $o b /+$ or $o b / o b$ mice as described previously [30]. The $o b / o b$ mice were also i.p. injected with vehicle ( $1 \%$ [vol./vol.] dimethylsulphoxide [DMSO] in PBS) or RG108 (Cayman, 13302; $6 \mathrm{mg} \mathrm{kg}^{-1}$ day $^{-1}$ ) daily for 5 weeks. The experimenters were not blind to group assignment and outcome assessment.

Real-time PCR assay The expression of mRNA and microRNA was quantified by real-time PCR using a LightCycler480 II Sequence Detection System (Roche, Basel, Switzerland). For further details, please refer to the ESM Methods.

Western blot analysis Total protein was isolated from HepG2 cells, primary mouse hepatocytes and liver tissue from mice. For further details, please refer to the ESM Methods.

DNA methylation analyses Genomic DNA was subjected to sodium bisulphite treatment using Methylamp (Epigentek, Brooklyn, NY, USA). The methylation status of the miR-9-3 promoter regions in hepatocytes was measured by methylationspecific PCR (MSP). Bisulphite genomic sequencing analyses were determined as previously described [31]. Quantitative MSP (qMSP) for the miR-9-3 promoter was performed using a real-time PCR assay as previously described [32]. For further details, please refer to the ESM Methods.

\section{Chromatin immunoprecipitation assays and chromatin} immunoprecipitation-quantitative PCR assay Chromatin immunoprecipitation (ChIP) and ChIP-quantitative real-time PCR (ChIP-qPCR) assays were performed using lysates of hepatocytes as described previously [33]. For further details, please refer to the ESM Methods.

Oligonucleotides and cell transfection MiR-9 was downregulated or upregulated by transfection with a miR-9 inhibitor or miR-9 mimic, respectively, using Lipofectamine 2000 (Invitrogen Life Technologies, Grand Island, NY, USA) according to the manufacturer's recommendations. All oligonucleotides were transfected at a final concentration of $100 \mathrm{nmol} / \mathrm{l}$. For further details, please refer to the ESM Methods.

Small interfering RNA Small interfering RNAs (siRNAs) specific for FOXO1 (si-Foxol) and control siRNA (si-NC) were synthesised (Ribobio, Guangzhou, China) and transfected using Lipofectamine 2000 into HepG2 cells. The sequences of si-Foxol and all other siRNAs are available in ESM Table 1.
Glucose tolerance test, insulin tolerance test and pyruvate tolerance test The glucose tolerance test (GTT) was performed by an i.p. injection of D-glucose $(2 \mathrm{~g} / \mathrm{kg}$ body weight, i.p.) after a $16 \mathrm{~h}$ overnight fast. Blood glucose levels were measured at $0,30,60,90$ and $120 \mathrm{~min}$ after injection. The insulin tolerance test (ITT), mice were fasted for $6 \mathrm{~h}$ and then injected with human insulin (Novo-Nordisk, Bagsværd, Denmark) at $0.75 \mathrm{U} / \mathrm{kg}$ body weight. Blood glucose levels were measured at $0,15,30$, and $60 \mathrm{~min}$. A pyruvate tolerance test (PTT) was performed to estimate gluconeogenesis. Mice were starved for $16 \mathrm{~h}$ and then injected i.p. with pyruvate $(2 \mathrm{~g} / \mathrm{kg}$ body weight, i.p.) dissolved in saline $(154 \mathrm{mmol} / \mathrm{l}$ $\mathrm{NaCl}$ ). Blood glucose levels were measured in the tail blood every $30 \mathrm{~min}$ for $2 \mathrm{~h}$. Blood glucose levels were measured using the hexokinase method (Thermo Fisher Scientific, Lafayette, CO, USA). Serum insulin levels were determined using an insulin ELISA kit (Lynco Research, St Charles, MO, USA).

Statistical analysis Statistical analyses were performed using SPSS 13.0 statistical analysis software (SPSS, Chicago, IL, USA). Data were expressed as the mean \pm SD. Analysis of variance (ANOVA) was used to determine the statistical differences among the groups. A $p$ value $<0.05$ was considered statistically significant. Results are representative of three individual experiments. No data, samples or animals were excluded or omitted from reporting.

\section{Results}

Hepatic miR-9 expression is decreased in obese mice and in normal mice under fasting conditions We evaluated the role of miR-9 in IR by determining the relative expression of miR-9 in two different obese mouse models. First, we found that the expression of mature miR-9 was clearly inhibited in livers from obese mice fed an HFD when compared with lean mice fed a normal chow diet (NCD) (Fig. 1a). Since mature miR-9 is encoded in mammals by three genes (miR-9-1, miR-9-2 and miR-9-3), we next measured the expression of miR-9-1, miR9-2 and miR-9-3. The level of miR-9-3 was significantly reduced in the HFD-fed mice, while the levels of miR-9-1 and miR-9-2 were not altered (Fig. 1b-d). Next, we observed a significant decrease in mature miR-9 and miR-9-3 expression in livers from $o b / o b$ obese mice when compared with expression in wild-type mouse livers (Fig. 1e, f). In addition, we measured hepatic miR-9 expression levels in C57BL/6J normal mice, following a $16 \mathrm{~h}$ fast. Interestingly, fasting reduced the miR-9 expression (Fig. 1g). We demonstrated, among the three isoforms of miR-9, that the level of miR-9-3 was the most highly expressed, followed by miR-9-1 and miR-9-2 in primary hepatocytes isolated from $\mathrm{C} 57 \mathrm{BL} / 6 \mathrm{~J}$ normal mice, which was confirmed by gel electrophoresis (ESM Fig. 1). 

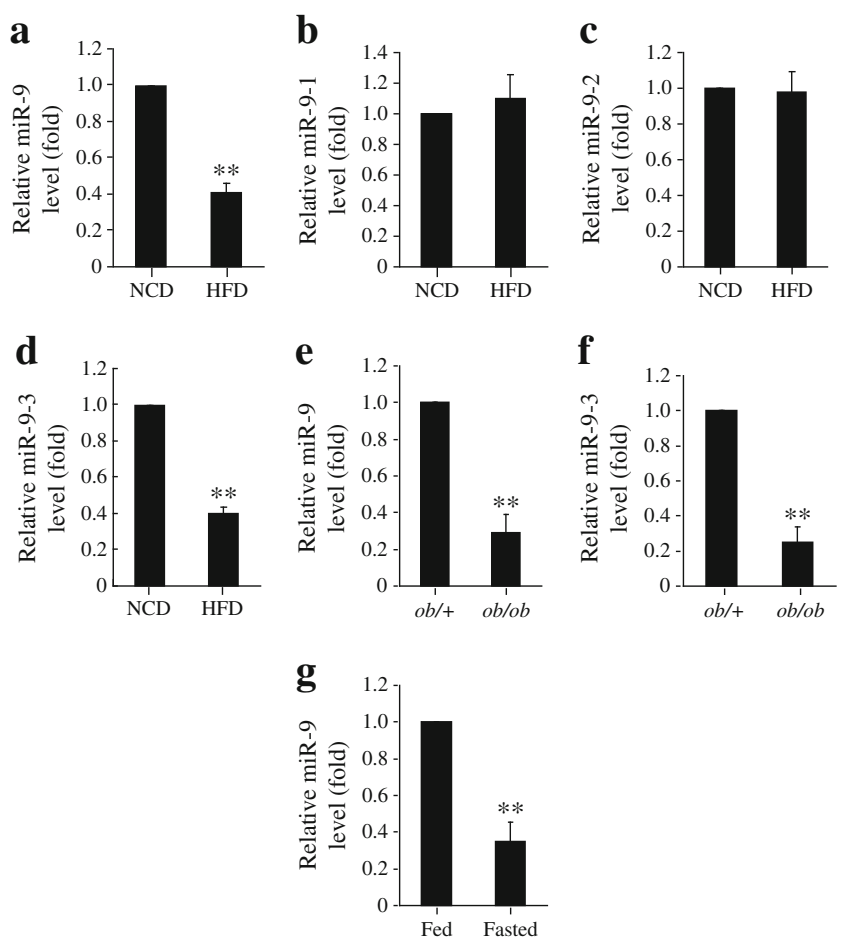

Fig. 1 Hepatic miR-9 expression is decreased in obese mice and in normal mice under fasting conditions. (a) Expression of mature miR-9 was measured in livers of lean mice fed an NCD and obese mice fed an $\operatorname{HFD}(n=3$ per group). Expression of miR-9-1 (b), miR-9-2 (c) and miR9-3 (d) was measured in livers of NCD-fed and HFD-fed mice $(n=3$ per group). Expression of mature miR-9 (e) and miR-9-3 (f) was measured in livers of $o b /+$ and $o b / o b$ mice ( $n=3$ per group). Expression of mature miR-9 (g) was measured in livers of fasted C57BL/6J normal mice (mice were fasted for $16 \mathrm{~h}$ ) and fed $\mathrm{C} 57 \mathrm{BL} / 6 \mathrm{~J}$ normal mice ( $n=3$ per group). $* * p<0.01$ vs control mice or $o b /+$ mice

DNA hypermethylation reduces hepatic miR-9 expression We next investigated if epigenetic DNA modification was a contributor to the silencing of miR-9 expression in our system by first treating cells with 5 -Aza-CdR, a specific inhibitor of DNA methylation, and with trichostatin A (TSA), a histone deacetylase inhibitor. We found that 5 -Aza-CdR significantly upregulated mature miR-9 expression in HepG2 cells and primary hepatocytes (Fig. 2a). However, TSA had no effect on miR-9 expression (Fig. 2b).

Interestingly, the miR-9-3 promoter was highly methylated in hepatocytes from HFD-fed mice when compared with those from NCD-fed mice. Likewise, the miR-9-3 promoter was hypermethylated in hepatocytes from $o b / o b$ mice (Fig. 2c). The qMSP results confirmed that the methylation of the miR-9-3 promoter was significantly increased in hepatocytes from HFD-fed and $o b / o b$ mice (Fig. 2d). However, methylation of the miR-9-1 and $m i R-9-2$ promoter was not significantly increased in hepatocytes from HFD-fed and $o b / o b$ mice compared with that in the lean mice (ESM Fig. 2).

DNMT1 governs hepatic miR-9 levels in $o b / o b$ mice DNA methylation is catalysed by DNA methyltransferase enzymes,
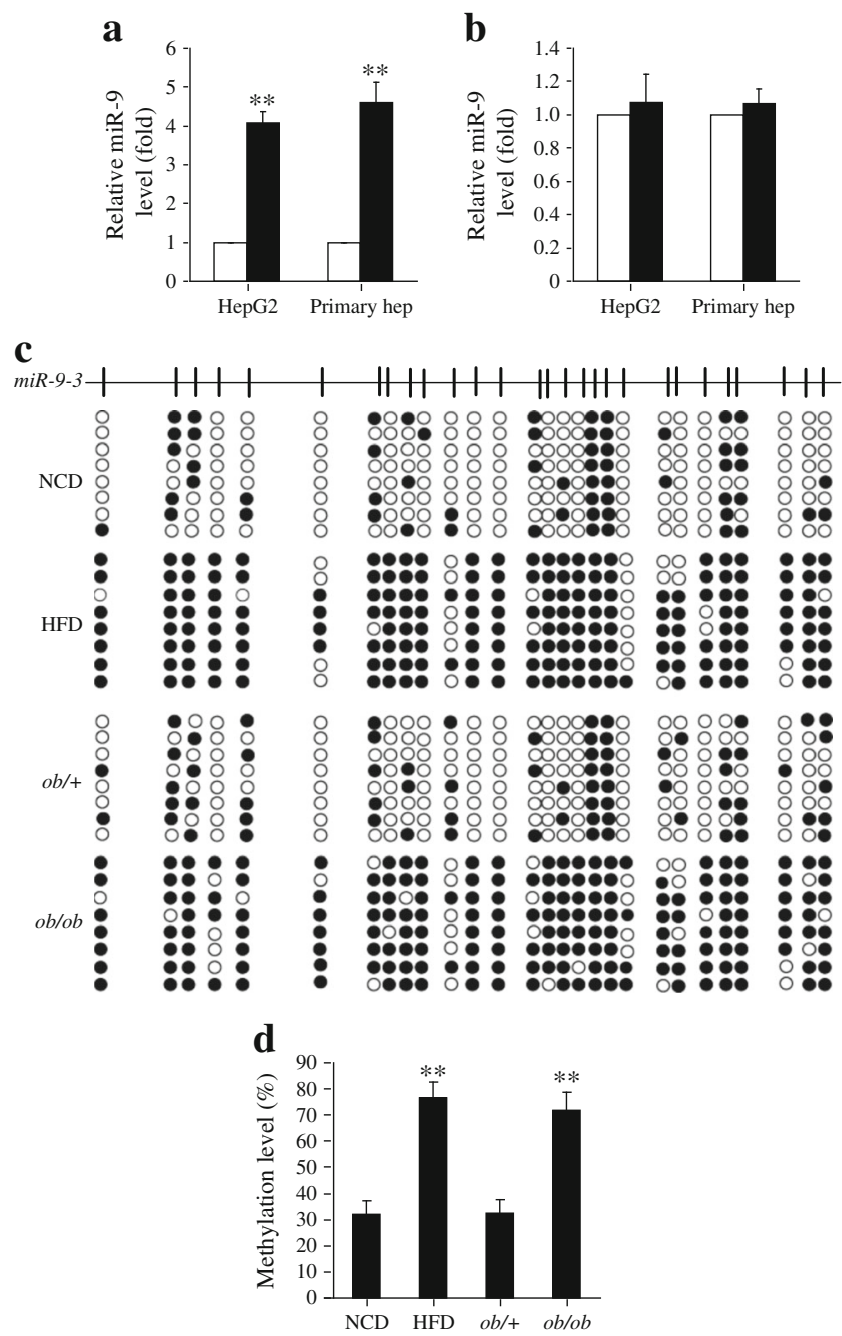

Fig. 2 DNA hypermethylation reduces hepatic miR-9 expression. (a) HepG2 cells and primary mouse hepatocytes were treated with (black bars) or without (white bars) 5 -Aza-CdR $(5 \mu \mathrm{mol} / \mathrm{l})$ for $48 \mathrm{~h}$ and then miR-9 expression was measured. (b) HepG2 cells and primary mouse hepatocytes were treated with (black bars) or without (white bars) the histone deacetylase inhibitor TSA (100 nmol/l) for $48 \mathrm{~h}$ and then miR-9 expression was measured. (c) Methylation levels of the miR-9-3 promoter were measured by bisulphite sequencing in hepatocytes from NCD-fed, HFD-fed, $o b /+$ and $o b / o b$ mice. (d) Quantification of $m i R-9-3$ promoter methylation was analysed in hepatocytes from NCD-fed, HFD-fed, $o b /+$ and $o b / o b$ mice ( $n=3$ per group). Values are means \pm SD and are representative of three individual experiments. ${ }^{* *} p<0.01$ vs control

which include DNMT1, DNMT3a and DNMT3b [34]. Quantitative ChIP (qChIP) assays demonstrated the association of DNMT1, DNMT3a and DNMT3b with the miR-9-3 promoter region. The binding of DNMT1, but not DNMT3a and DNMT3b, onto the promoters of miR-9-3 was increased in hepatocytes from $o b / o b$ mice when compared with those from lean mice (Fig. 3a-c). The DNMT inhibitor RG108, when i.p. injected into $o b / o b$ mice, had no effect on body weight, liver function or mortality (data not shown), but induced an obvious increase in miR-9 expression in the liver when compared with the expression in vehicle-injected $o b / o b$ mice (Fig. 3d). 

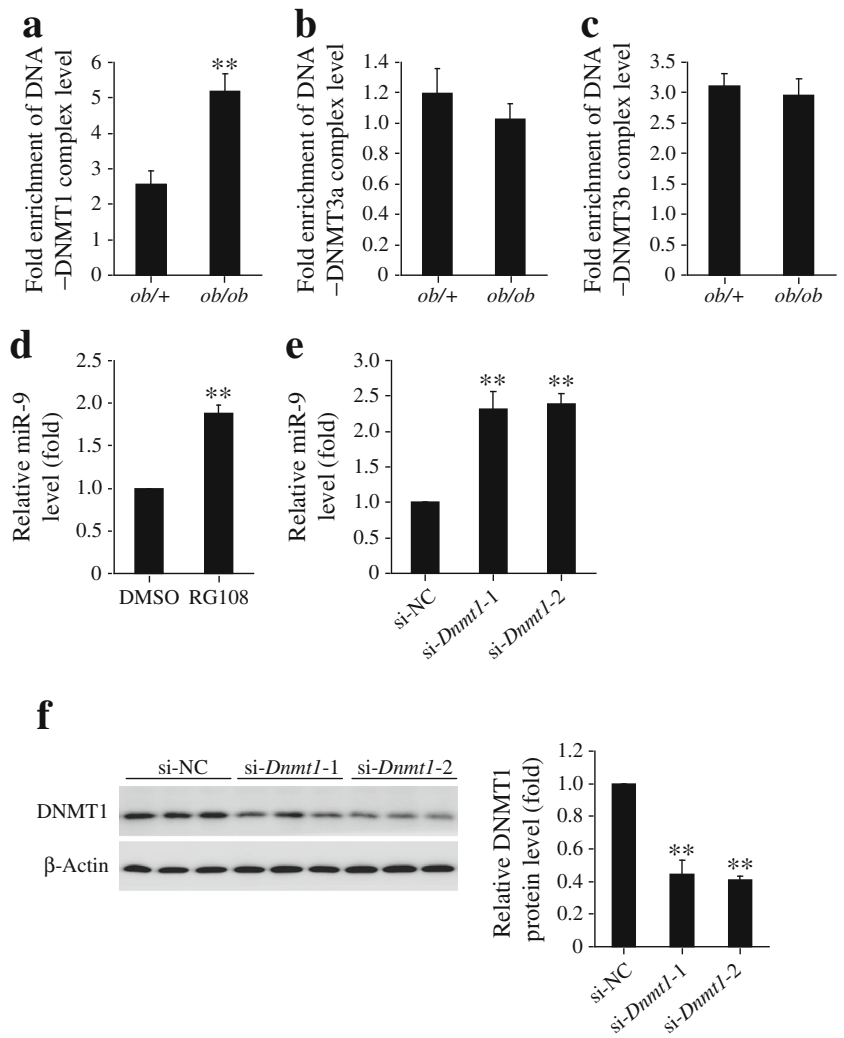

Fig. 3 DNMT1 governs hepatic miR-9 levels in $o b / o b$ mice. ChIP-qPCR analysis of the capacity for DNMT1 (a), DNMT3a (b) and DNMT3b (c) binding to the miR-9-3 promoter was performed in hepatocytes from $o b /+$ and $o b / o b$ mice. Values are means $\pm \mathrm{SD}$ and are representative of three individual experiments. (d) The $o b / o b$ mice were i.p. injected with vehicle (1\% DMSO) or RG108 $\left(6 \mathrm{mg} \mathrm{kg}^{-1} \mathrm{day}^{-1}\right)$ for 5 weeks $(n=7$ for each group). The expression of miR-9 was measured in hepatocytes from $o b /$ $o b$ mice. The $o b / o b$ mice were injected with si-NC, si-Dnmt1-1 and siDnmt1-2 daily for 30 days via the tail vein and then miR-9 (e) and DNMT1-1 (f) expression in the liver was determined ( $n=7$ for each group). ${ }^{* *} p<0.01$ vs $o b /+$ mice or vehicle or si-NC

We also measured the effect of DNMT1 deletion on miR-9 expression in $o b / o b$ mice. The miR-9 level was significantly increased in hepatocytes from $o b / o b$ mice following injection of si-Dnmt1 (Fig. 3e). Western blot data demonstrated that siDnmt1-1 and si-Dnmt1-2 silenced DNMT1 protein levels in hepatocytes from $o b / o b$ mice (Fig. 3f). These results indicated that DNMT1 decreased miR-9 expression through enhancement of promoter methylation.

Knockdown of DNMT1 alleviates the palmitate-induced decrease of miR-9 expression in vitro We examined the potential involvement of DNMT1 in the in vitro expression of miR-9. Treatment of primary mouse hepatocytes with palmitate significantly decreased miR-9 expression while clearly increasing miR-9-3 promoter methylation. These effects could be rescued by deletion of DNMT1 (Fig. 4a, b). DNMT1 knockdown led to a significant decrease in DNMT1 binding to the miR-9-3 promoter in palmitateexposed hepatocytes (Fig. 4c).
Overexpression of hepatic miR-9 suppresses gluconeogenesis in obese mice We investigated whether the increase in miR-9 expression in obese mice livers has any effect on glycaemic control in obese mice. In the HFD-fed mice, upregulation of miR-9 expression in the liver decreased the random and fasting blood glucose levels (Fig. 5a). The PTT results showed that miR-9 overexpression in the liver significantly decreased the conversion of pyruvate to glucose in the HFD-fed mice, suggesting a decrease in gluconeogenesis (Fig. 5b). Interestingly, the GTT results showed that increased hepatic miR-9 expression improved glucose tolerance in HFD mice (Fig. 5c). The ITT results also indicated that miR-9 overexpression improved insulin sensitivity (Fig. 5d). Gene expression analysis showed a significant increase of miR-9 expression (Fig. 5e), and a significant decrease of FOXO1, PEPCK and G6Pase protein levels (Fig. 5f) in the isolated hepatocytes from HFD-fed mice with miR-9 overexpression. Similar results were also obtained in miR-9-overexpressing ob/ob mice (ESM Fig. 3a-c). These results indicated that miR-9 acted as a regulator of hepatic gluconeogenesis and glycaemic control in vivo.

Deletion of hepatic miR-9 leads to an increase of fasting blood glucose levels in lean mice We further investigated whether hepatic miR-9 affects insulin sensitivity by injecting an miR-9 inhibitor into $\mathrm{C} 57 \mathrm{BL} / 6 \mathrm{~J}$ mice via the tail vein, as previously described [29]. Figure 6a shows that the miR-9 inhibitor significantly decreased miR-9 expression in hepatocytes. Deletion of hepatic miR-9 also elevated random and fasting blood glucose levels (Fig. 6b). The PTT results showed that downregulation of miR-9 expression increased the conversion of pyruvate to glucose in lean mice (Fig. 6c). The GTT data showed impairment of glucose tolerance in response to decreased miR-9 expression in lean mouse livers (Fig. 6d), while the ITT data indicated suppression of systemic insulin sensitivity (Fig. 6e). In addition, inhibition of miR-9 expression led to a significant increase in the protein levels of FOXO1, PEPCK and G6Pase (Fig. 6f).

MiR-9 suppresses gluconeogenesis and HPG by directly targeting FOXO1 The transcription factor FOXO1 is a regulator of gluconeogenesis and is a target of miR-9 in haematopoietic cells and neural cells [21,35]. We investigated whether FOXO1 is required for the miR-9 inhibition of gluconeogenesis and glucose output. Primary hepatocytes were transfected with miR-9 inhibitor and si-NC or si-Foxo1. Figure 7 a shows a significant elevation of FOXO1 expression in cells transfected with miR-9 inhibitor; this effect was reversed by transfection with si-Foxol. The increases of PEPCK and G6Pase expression by miR-9 deletion were partially abolished in hepatocytes by silencing of FOXO1 expression (Fig. 7b). FOXO1 knockdown completely reversed the glucose production in hepatocytes treated with miR-9 inhibitor 

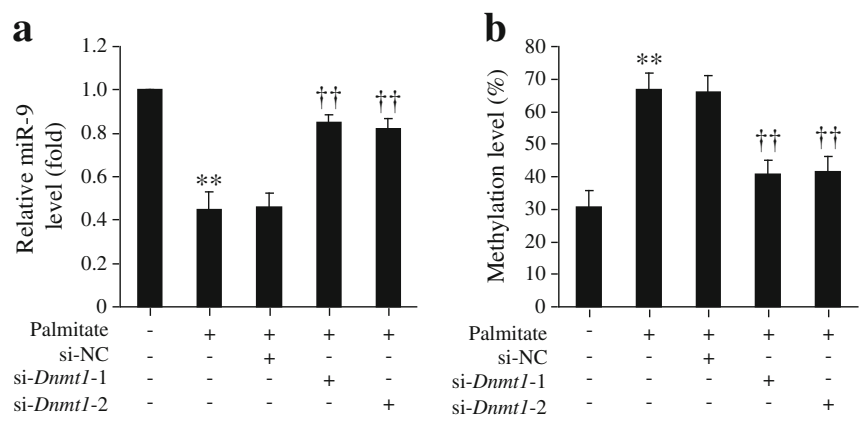

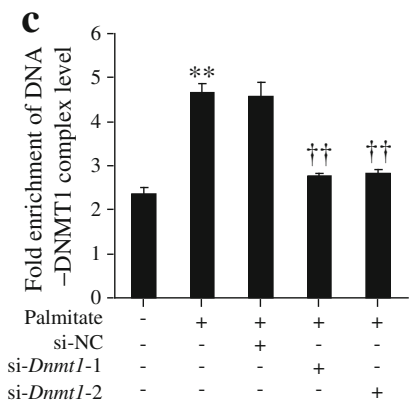

of miR-9, (b) the degree of miR-9-3 promoter methylation and (c) the capacity of DNMT1 binding to miR-9-3 promoter were measured. Values are means $\pm \mathrm{SD}$ and are representative of three individual experiments. $* * p<0.01$ vs control; ${ }^{\dagger \dagger} p<0.01$ vs palmitate + si-NC
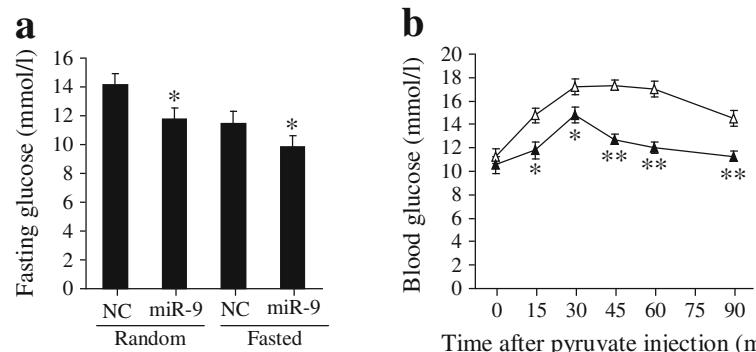

Time after pyruvate injection ( $\mathrm{min})$

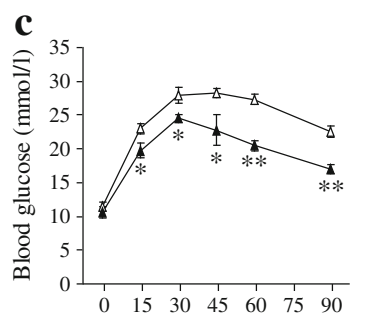

Time after glucose injection ( $\mathrm{min})$

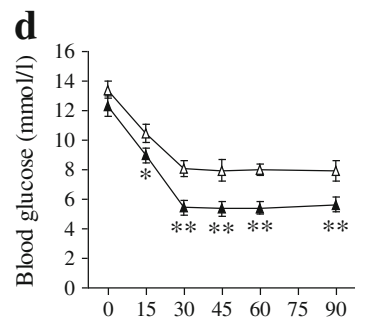

Time after insulin injection ( $\mathrm{min})$
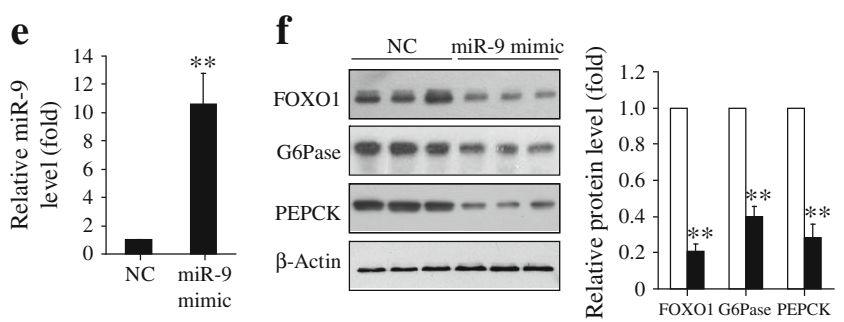

Fig. 5 Overexpression of hepatic miR-9 improves insulin sensitivity in obese mice. Mice were fed an HFD for 15 weeks, and then were injected with NC or miR-9 mimic daily for 30 days via the tail vein. (a) Random and fasting blood glucose levels were measured ( $n=7$ per group). (b) PTT was performed in mice fasted for $16 \mathrm{~h}(n=7$ per group; $\mathrm{NC}$, white triangles; miR-9 mimic, black triangles). (c) GTT was performed in mice fasted for $16 \mathrm{~h}(n=7$ per group; $\mathrm{NC}$, white triangles; miR-9 mimic, black triangles). (d) ITT was performed in mice fasted for $4 \mathrm{~h}(n=7$ per group; NC, white triangles; miR-9 mimic, black triangles). (e) The expression of miR-9 in hepatocytes from HFD-fed mice was measured $(n=3$ per group). (f) The protein levels of FOXO1, PEPCK and G6Pase were determined ( $n=3$ per group; NC, white bars; miR-9 mimic, black bars). Values are means $\pm \mathrm{SD}$ and are representative of three individual experiments. ${ }^{*} p<0.05, * * p<0.01$ vs NC group
(Fig. 7c). These data suggested that miR-9 inhibited gluconeogenesis and glucose production by regulating FOXO1.

\section{Discussion}

Growing evidence now indicates that miRs play key roles in the development of IR and diabetes [36]. This study revealed, for the first time, that reduced hepatic miR-9 levels contribute to gluconeogenesis and IR in HFD mice and $o b / o b$ mice. MiR-9 may have alternative and non-overlapping functions in different cell types. In pancreatic beta cells, miR-9 has been proposed to negatively regulate insulin secretion [20]. MiR-9 acted as an oncogene in leukaemia [37], but as a tumour suppressor in hepatocellular carcinoma [38]. It is worth mentioning that we have not observed significant differences in body weight, plasma alanine transaminase (ALT) and aspartate transaminase (AST) levels and mortality of mice injected with miR-9 inhibitor or miR-9 mimic compared with mice injected with NC (data not shown). This indicated that miR-9 might play a direct role in suppression of glucose production in liver. Therefore, enhancing hepatic miR-9 expression may be a potential pharmacological strategy to improve insulin sensitivity.

MiR-9 has been found to be relevant to the differentiation of normal hepatocytes [39]. Data from the present study show that miR-9 expression was significantly decreased in obese mouse livers. In mice and humans, mature miR-9 is encoded by three genes: miR-9-1, miR-9-2 and miR-9-3. Here, we demonstrated that, of these, miR-9-3 is the one predominantly expressed in hepatocytes. Interestingly, a decrease in miR-9-3 expression was also observed in the obese mouse liver. Taken together, these data suggest that miR-9-3 is specifically inactivated in the fatty liver, resulting in downregulation of mature miR-9 expression in the obese mouse liver.

How miR-9 is deregulated in the obese mouse liver is not clear. Previous studies reported that DNA methylation is involved in regulating miR-9 expression in haematopoietic cells 

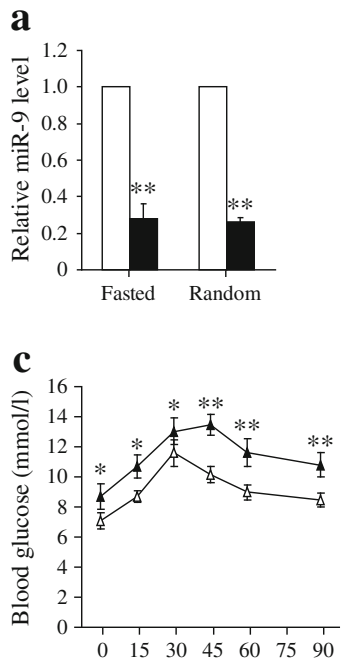

Time after pyruvate injection ( $\mathrm{min})$

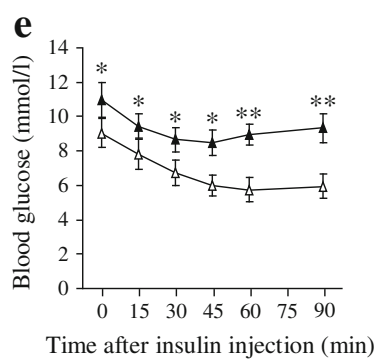

a
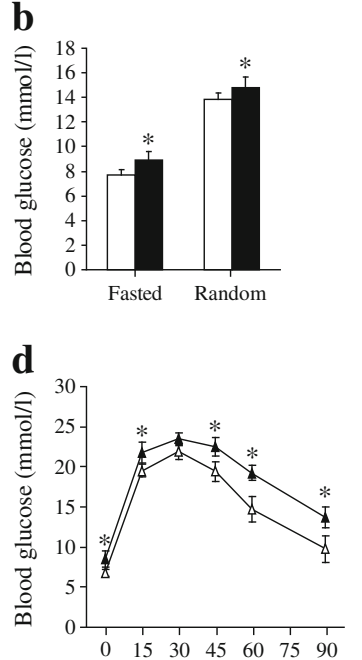

Time after glucose injection ( $\mathrm{min})$

f

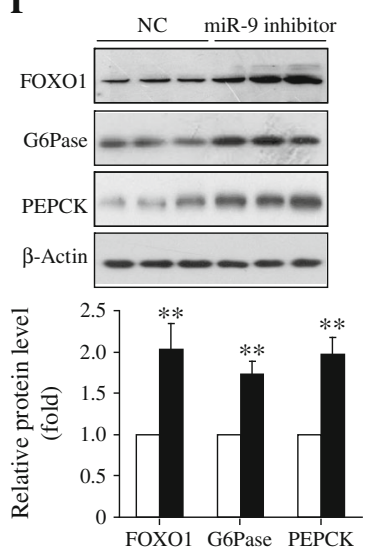

Fig. 6 Deletion of hepatic miR-9 leads to an increase in fasting blood glucose levels in lean mice. C57BL/6J male mice (8-week-old) were injected with $\mathrm{NC}$ (white bars) or miR-9 inhibitor (black bars) daily for 30 days via the tail vein. (a) The expression of miR-9 was measured in hepatocytes from C57BL/6J mice ( $n=3$ per group). (b) Random and fasting blood glucose levels were measured ( $n=7$ per group). (c) PTT was performed in mice fasted for $16 \mathrm{~h}(n=7$ per group). (d) GTT was performed in mice fasted for $16 \mathrm{~h}$ ( $n=7$ each group). (e) ITT was performed in mice fasted for $4 \mathrm{~h}$ ( $n=7$ per group). (c-e) $\mathrm{NC}$, white triangles; miR-9 inhibitor, black triangles. (f) The protein levels of FOXO1, PEPCK and G6Pase were determined ( $n=3$ per group). ${ }^{*} p<0.05$, $* * p<0.01$ vs NC group

[21]. Here we showed that hepatocytes isolated from HFD-fed and $o b / o b$ mice show an increase in DNA methylation at the $m i R-9$ promoter, concomitantly with the enhanced accumulation of DNMT1 in the miR-9-3 promoter. More importantly, the DNMT1 inhibitor reversed the decrease in miR-9 expression in hepatocytes from obese mice. In addition, silencing of DNMT1 alleviated the palmitate-induced decrease in miR-9 expression in primary mouse hepatocytes. Collectively, these findings clearly demonstrated a role for DNA methylation in regulating obesity-induced downregulation of hepatic miR-9 expression.

One question that arises from our results is the precise genes that are regulated by miR-9. The transcription factor

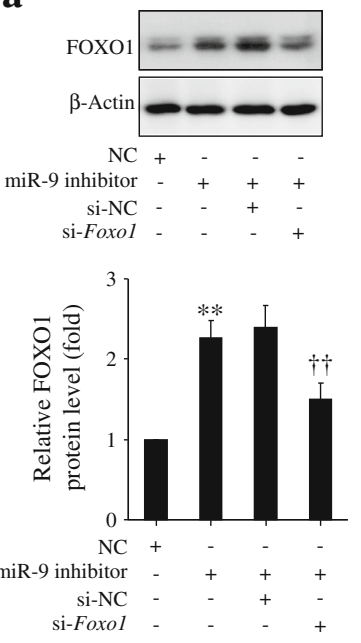

b

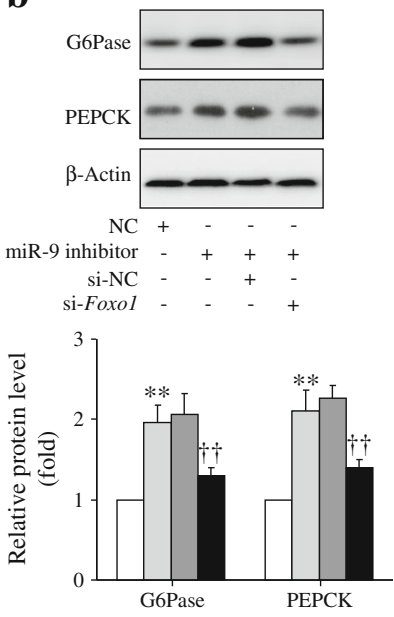

c

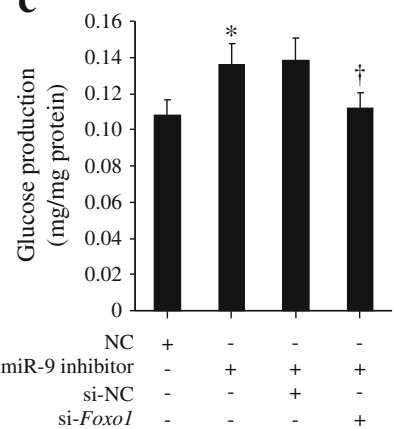

Fig. 7 MiR-9 suppressed gluconeogenesis and HPG by direct targeting of FOXO1. Primary mouse hepatocytes were transfected with miR-9 inhibitor and si-NC or si-Foxol for $48 \mathrm{~h}$. The expression of FOXO1 (a), PEPCK and G6Pase (b) was measured. (c) Glucose production in hepatocytes was determined. (NC, white bars; miR-9 inhibitor, light grey bars; miR-9 inhibitor + si-NC, dark grey bars; miR- 9 inhibitor + si-Foxol, black bars). ${ }^{*} p<0.05,{ }^{* *} p<0.01$ vs NC; ${ }^{\dagger} p<0.05,{ }^{\dagger} p<0.01$ vs miR-9 inhibitor + si-NC

FOXO1 has been identified as a target of miR-9 in haematopoietic cells and neural cells [21,35]. We found that a miR-9 mimic significantly inhibited the activity of the 3'untranslated region of Foxol mRNA and FOXO1 expression in mouse liver cells (data not shown). Given that FOXO1 is an important regulator of hepatic gluconeogenesis [6, 7], we further speculated that FOXO1 mediates miR-9 function in the regulation of gluconeogenesis and HGP in hepatocytes. This speculation was supported by the observation that silencing of FOXO1 expression could almost completely reverse the increase in gluconeogenic enzyme expression and the glucose output in hepatocytes induced by miR-9 deletion.

In conclusion, our study demonstrates that DNA hypermethylation contributes to downregulation of miR-9 expression in the obese mouse liver, leading to upregulation of FOXO1 expression, and finally to inappropriately activated gluconeogenesis. These findings provide valuable insights into the molecular mechanisms responsible for glucose intolerance and IR. 
Acknowledgements The authors would like to thank F. Chen and C. Dai, Nanjing Medical University, for valuable discussion.

Funding This work was supported by the National Natural Science Foundation of China Grants 31271263/81470040 to XL; National Basic Research Program of China (2011CB504000) and National Natural Science Foundation of China Grants 81070656/81170252 to DS.

Duality of interest The authors declare that there is no duality of interest associated with this manuscript.

Contribution statement All authors took part in the conception and design of the study, as well as either drafting or critically revising the manuscript. All authors have approved the final version of the manuscript. CY, JC, ML, WX, YH, HY, DS and NC collected the data and carried out the data analysis. XL is responsible for the integrity of the work as a whole.

\section{References}

1. Altaf QA, Barnett AH, Tahrani AA (2015) Novel therapeutics for type 2 diabetes: insulin resistance. Diabetes Obes Metab 17:319-334

2. Vozarova B, Stefan N, Lindsay RS et al (2002) High alanine aminotransferase is associated with decreased hepatic insulin sensitivity and predicts the development of type 2 diabetes. Diabetes 51:18891895

3. Kowalski GM, Bruce CR (2014) The regulation of glucose metabolism: implications and considerations for the assessment of glucose homeostasis in rodents. Am J Physiol Endocrinol Metab 307: e859-e871

4. Mutel E, Gautier-Stein A, Abdul-Wahed A et al (2011) Control of blood glucose in the absence of hepatic glucose production during prolonged fasting in mice: induction of renal and intestinal gluconeogenesis by glucagon. Diabetes 60:3121-3131

5. Kousteni S (2011) FoxO1: a molecule for all seasons. J Bone Miner Res 26:912-917

6. Rui L (2014) Energy metabolism in the liver. Compr Physiol 4: 177-197

7. Oh KJ, Han HS, Kim MJ, Koo SH (2013) CREB and FoxO1: two transcription factors for the regulation of hepatic gluconeogenesis. BMB Rep 46:567-574

8. Valenti L, Rametta R, Dongiovanni P et al (2008) Increased expression and activity of the transcription factor FOXO1 in nonalcoholic steatohepatitis. Diabetes 57:1355-1362

9. Qu S, Altomonte J, Perdomo G et al (2006) Aberrant forkhead box O1 function is associated with impaired hepatic metabolism. Endocrinology 147:5641-5652

10. Nakae J, Biggs WH 3rd, Kitamura T et al (2002) Regulation of insulin action and pancreatic beta-cell function by mutated alleles of the gene encoding forkhead transcription factor Foxo1. Nat Genet 32:245-253

11. Zhang K, Li L, Qi Y et al (2012) Hepatic suppression of Foxol and Foxo3 causes hypoglycemia and hyperlipidemia in mice. Endocrinology 153:631-646

12. Matsumoto M, Pocai A, Rossetti L, Depinho RA, Accili D (2007) Impaired regulation of hepatic glucose production in mice lacking the forkhead transcription factor Foxol in liver. Cell Metab 6:208-216

13. Kitamura T (2013) The role of FOXO1 in $\beta$-cell failure and type 2 diabetes mellitus. Nat Rev Endocrinol 9:615-623
14. Costa FF (2005) Non-coding RNAs: new players in eukaryotic biology. Gene 357:83-94

15. Kadamkode V, Banerjee G (2014) Micro RNA: an epigenetic regulator of type 2 diabetes. Microrna 3:86-97

16. Wang B, Hsu SH, Frankel W, Ghoshal K, Jacob ST (2012) Stat3mediated activation of microRNA-23a suppresses gluconeogenesis in hepatocellular carcinoma by down-regulating glucose-6phosphatase and peroxisome proliferator-activated receptor gamma, coactivator 1 alpha. Hepatology 56:186-197

17. Liang J, Liu C, Qiao A et al (2013) MicroRNA-29a-c decrease fasting blood glucose levels by negatively regulating hepatic gluconeogenesis. J Hepatol 58:535-542

18. Li K, Zhang J, Yu J et al (2015) MicroRNA-214 suppresses gluconeogenesis by targeting activating transcriptional factor 4. J Biol Chem 290:8185-8195

19. Ramachandran D, Roy U, Garg S, Ghosh S, Pathak S, KolthurSeetharam U (2011) Sirt1 and mir-9 expression is regulated during glucose-stimulated insulin secretion in pancreatic $\beta$-islets. FEBS J 278:1167-1174

20. Plaisance V, Abderrahmani A, Perret-Menoud V, Jacquemin P, Lemaigre F, Regazzi R (2006) MicroRNA-9 controls the expression of granuphilin/Slp4 and the secretory response of insulinproducing cells. J Biol Chem 281:26932-26942

21. Senyuk V, Zhang Y, Liu Y et al (2013) Critical role of miR-9 in myelopoiesis and EVI1-induced leukemogenesis. Proc Natl Acad Sci U S A 110:5594-5599

22. Kirchner H, Osler ME, Krook A, Zierath JR (2013) Epigenetic flexibility in metabolic regulation: disease cause and prevention? Trends Cell Biol 23:203-209

23. Bastien-Dionne PO, Valenti L, Kon N, Gu W, Buteau J (2011) Glucagon-like peptide 1 inhibits the sirtuin deacetylase SirT1 to stimulate pancreatic $\beta$-cell mass expansion. Diabetes 60:32173222

24. Feil R, Fraga MF (2011) Epigenetics and the environment: emerging patterns and implications. Nat Rev Genet 13:97-109

25. Ma Y, Xia W, Wang DQ et al (2013) Hepatic DNA methylation modifications in early development of rats resulting from perinatal BPA exposure contribute to insulin resistance in adulthood. Diabetologia 56:2059-2067

26. Jiang M, Zhang Y, Liu M et al (2011) Hypermethylation of hepatic glucokinase and L-type pyruvate kinase promoters in high-fat dietinduced obese rats. Endocrinology 152:1284-1289

27. Nguyen CT, Gonzales FA, Jones PA (2001) Altered chromatin structure associated with methylation-induced gene silencing in cancer cells: correlation of accessibility, methylation, MeCP2 binding and acetylation. Nucleic Acids Res 29: $4598-4606$

28. Edwards M, Houseman L, Phillips IR, Shephard EA (2013) Isolation of mouse hepatocytes. Methods Mol Biol 987:283-293

29. Wu H, Ng R, Chen X, Steer CJ, Song G (2015) MicroRNA-21 is a potential link between non-alcoholic fatty liver disease and hepatocellular carcinoma via modulation of the HBP1-p53-Srebp1c pathway. Gut. doi:10.1136/gutjnl-2014-308430

30. Kim AY, Park YJ, Pan X et al (2015) Obesity-induced DNA hypermethylation of the adiponectin gene mediates insulin resistance. Nat Commun 6:7585

31. Lujambio A, Calin GA, Villanueva A et al (2008) A microRNA DNA methylation signature for human cancer metastasis. Proc Natl Acad Sci U S A 105:13556-13561

32. Papathanasiou I, Kostopoulou F, Malizos KN, Tsezou A (2015) DNA methylation regulates sclerostin (SOST) expression in osteoarthritic chondrocytes by bone morphogenetic protein 2 (BMP-2) induced changes in Smads binding affinity to the $\mathrm{CpG}$ region of SOST promoter. Arthritis Res Ther 17:160

33. Lin HY, Yin Y, Zhang JX et al (2012) Identification of direct forkhead box $\mathrm{O} 1$ targets involved in palmitate-induced apoptosis in 
clonal insulin-secreting cells using chromatin immunoprecipitation coupled to DNA selection and ligation. Diabetologia 55:2703-2712

34. Jurkowska RZ, Jurkowski TP, Jeltsch A (2011) Structure and function of mammalian DNA methyltransferases. Chembiochem 12:206-222

35. Kim DY, Hwang I, Muller FL, Paik JH (2015) Functional regulation of FoxO1 in neural stem cell differentiation. Cell Death Differ 22:2034-2045

36. Fernandez-Valverde SL, Taft RJ, Mattick JS (2011) MicroRNAs in $\beta$-cell biology, insulin resistance, diabetes and its complications. Diabetes 60:1825-1831
37. Chen P, Price C, Li Z et al (2013) miR-9 is an essential oncogenic microRNA specifically overexpressed in mixed lineage leukemiarearranged leukemia. Proc Natl Acad Sci U S A 110:11511-11516

38. Zhang J, Cheng J, Zeng Z et al (2015) Comprehensive profiling of novel microRNA-9 targets and a tumor suppressor role of microRNA-9 via targeting IGF2BP1 in hepatocellular carcinoma. Oncotarget 6:42040-42052

39. Kim N, Kim H, Jung I et al (2011) Expression profiles of miRNAs in human embryonic stem cells during hepatocyte differentiation. Hepatol Res 41:170-183 